\title{
Exactly solvable Hamiltonians in quantum computing
}

\section{Grigori Giorgadze*}

Institute of Cybernetics,

5 Sandro Euli str., Tbilisi, Georgia

Tbilisi State University, Department of Mathematics

13 University str.,Tbilisi, Georgia

E-mail: gia.giorgadze@tsu.ge

In this paper, we investigate the problem of simulation of quantum-mechanical dynamical system with prescribed properties using of exactly solvable Hamiltonians. A method for solving timedependent matrix Schrödinger equations in an explicit analytical form will be developed. It is based on exactly solvable time-independent problems, a certain choice of the initial conditions, and special time-dependent gauge transformations converting time-independent problems to timedependent ones. An application of the exactly solvable time-dependent problems thus obtained to the construction of a universal set of gates for quantum computers is also presented.

RDP online workshop "Recent Advances in Mathematical Physics" - Regio2020,

5-6 December 2020

online

* Speaker 


\section{Construction of a Time-Dependent Hamiltonian}

In this section we give a brief overview of one possible method for obtaining exact solutions to evolution of quantum mechanical systems, including the cyclic evolution of quantum systems described by time-periodic Hamiltonians (for more details see [7], [14]). Many interesting phenomena, such as the molecular Aharonov-Bohm effect [11], geometric phase [2], [1], level crossing [9] identified with the Landau-Zener transitions [16], [10], and dynamical localization of particles in systems with a limited spatial dimension [13], have been revealed in atomic and molecular physics, quantum chemistry, quantum optics, and solid state physics [3]. Active investigations in quantum computers (see [12] and references therein) refreshed interest in the geometric phase phenomenon in quantum mechanics. In paper [15] was proposed a design of holonomic quantum computer on the basis of the non-Abelian Berry phase. For this reason, the problem of simulation of dynamical systems with prescribed properties by quantum-mechanical methods is of current interest.

Our approach, given here, is based on transformation of solvable time-independent equations into time-dependent ones by employing a set of special timedependent transformation operators. A class of periodic time-dependent Hamiltonians with cyclic solutions is constructed in closed analytic form and the non-adiabatic geometric phase is determined in terms of the obtained solutions.

The time evolution of quantum system is governed by the Schrödinger equation

$$
i \frac{d|\Psi(r, t)\rangle}{\partial t}=H(r, t)|\Psi(r, t)\rangle
$$

with $\hbar=1$ and $T$-periodic time-dependent Hamiltonian, $H(t)=H(t+T)$ and has the form

$$
H(r, t)=\widehat{p}_{r}^{2}+V(r, t)
$$

where $V(r, t)$ is a square time-periodic matrix such that $V(r, t+T)=V(r, t), \widehat{p}_{r}=-i \nabla_{r}$ is the angular momentum operator determining the kinetic energy.

It is known that a classical periodic system returns to its initial state after a period, whereas a quantum system acquires an additional Berry phase (see [4], [14]), which is the difference between the total and dynamic phases. The quantization of the Berry phase is associated with the quantization of the spin alignment and is important in our consideration for obtaining quantum gates.

We use the time-independent Hamiltonian $\widetilde{H}(r)$ and a unitary time-dependent transformation $\mathcal{G}(t)$

$$
|\Psi(r, t)\rangle=\mathcal{G}(t)|\Phi(r, t)\rangle,
$$

by means of which the known time-independent Hamiltonian is changed to the time-dependent one

$$
H(t)=\mathcal{G}(t) \widetilde{H} \mathcal{G}^{\dagger}(t)+i \dot{\mathcal{G}}(t) \mathcal{S}^{\dagger}(t)
$$

Here, $|\Phi(r, t)\rangle$ satisfies the equation of motion (1) with the time-independent Hamiltonian $\widetilde{H}(r)$ and it is of the form

$$
|\Phi(r, t)\rangle=\exp (-i \widetilde{H}(r) t)|\Phi(r, 0)\rangle
$$

a dot in expression (3) means a time-derivative. 
Clearly, the solutions $|\Psi(r, t)\rangle$ and $|\Phi(r, t)\rangle$ can be properly defined by solutions of the timeindependent problem

$$
\widetilde{H}|\Phi(\widetilde{\mathcal{E}})\rangle=\widetilde{\mathcal{E}}|\Phi(\widetilde{\mathcal{E}})\rangle .
$$

Note that if the system of equations (5) with some known time-independent Hamiltonian $\widetilde{H}(r)$ is exactly solvable, the system of equations (1) with the time-dependent Hamiltonian (3) admits exact solutions, too. The result depends on transformation operators $\mathcal{G}(t)$ and the choice of initial states.

As a particular case consider reconstruction of the $2 \times 2$ periodic time-dependent Hamiltonian taken in the form

$$
H(t, r)=\left(p_{r}^{2}+q(r)\right) \hat{I}+2(\mathbf{s} \cdot \mathbf{B}(r, t)) .
$$

We start with the time-independent Hamiltonian

$$
\widetilde{H}(r)=\left(p_{r}^{2}+q(r)\right) \hat{I}+2(\mathbf{s} \cdot \mathbf{B}(r)),
$$

By means of a unitary time-dependent transformation taken in the form

$$
\mathcal{G}(t)=\exp (-i \mathbf{s} \cdot \mathbf{h}(t))=\exp \left(-i \sum_{i=1}^{3} s_{i} h_{i}(t)\right),
$$

the time-independent Hamiltonian (7) with regard to equations (1) and (3) turns to the timedependent Hamiltonian

$$
H(r, t)=\left(p_{r}^{2}+q(r)\right) \hat{I}+2 \exp (-i \mathbf{s} \cdot \mathbf{h}(t))(\mathbf{s} \cdot \mathbf{B}(r)) \exp (i \mathbf{s} \cdot \mathbf{h}(t))
$$

Here $\mathbf{s}=(1 / 2) \boldsymbol{\sigma}$ is the spin operator, $\boldsymbol{\sigma}=\left(\hat{\sigma}_{1}, \hat{\sigma}_{2}, \hat{\sigma}_{3}\right)$ and $\hat{\sigma}_{i}$ are the Pauli matrices.

The solutions of (1) with Hamiltonian (9), according to (2) and (4), are represented as

$$
|\Psi(r, t)\rangle=\exp (-i \mathbf{s} \cdot \mathbf{h}(t) \exp (-i \widetilde{H}(r) t)|\Phi(r, t=0)\rangle .
$$

Obviously, the transformation (8) does not change the first term of (7) and transforms the second term. The Hamiltonians in the form of (6) and (9) can be used to describe the motion of a spin 1/2-particle in the space-nonuniform and time-dependent magnetic field or can be applied in investigating multi-level atoms and nuclei.

In terms of the evolution operator $\mathcal{U}(t)=\mathcal{U}(t, 0)$, the solution $|\Psi(r, t)\rangle$ is

$$
|\Psi(r, t)\rangle=\mathcal{U}(t)|\Psi(r, 0)\rangle, \quad \mathcal{U}(0)=1 .
$$

It is easy to see that from (10) and (11) follows an important relationship between the operators $\mathcal{U}(t)$ and $\mathcal{G}(t): \mathcal{U}(t)=\mathcal{G}(t) \exp (-i \widetilde{H} t) \mathcal{G}(0)$. In this case, if $|\Psi(r, 0)\rangle=|\Phi(r, 0)\rangle$, then

$$
\mathcal{U}(t)=\mathcal{G}(t) \exp (-i \widetilde{H} t)=\exp (-i \mathbf{s} \cdot \mathbf{h}(t)) \exp (-i \widetilde{H} t) .
$$

Let us now consider some particular examples of constructing time-dependent with corresponding solutions by using $S U(2)$ transformation (8) in which the components of $\mathbf{h}(t)$ are linear functions of time, $h_{i}(t)=\omega_{i} t$. Let $\mathcal{G}(t)$ be chosen as an operator of rotation around $z$-axis

$$
\mathcal{G}_{3}(t)=\exp \left(-i \hat{\sigma}_{3} \omega t / 2\right)=\left(\begin{array}{cc}
\exp (-i \omega t / 2) & 0 \\
0 & \exp (i \omega t / 2)
\end{array}\right),
$$


where $\omega$ is a constant angular velocity. Then, in accordance with (9), the time-independent Hamiltonian (7) is transformed into the time-dependent one as

$$
H(r, t)=\left(p_{r}^{2}+q(r)\right) \hat{I}+\left(\begin{array}{cc}
B_{3}(r)+\frac{\omega}{2} & B_{1}(r) \exp (-i \omega t) \\
B_{1}(r) \exp (i \omega t) & -B_{3}+\frac{\omega}{2}
\end{array}\right) .
$$

It is evident that $H(r, t)$ is $T$-periodic, $H(r, t=0)=H(r, t=T), T=2 \pi / \omega$. Its Routhian is $\widetilde{H}(r)$ connected with $H(r, t=0)$ by $H(r, 0)=\bar{H}(r)+\frac{\omega}{2} \hat{\sigma}_{3}$. With the use of identity

$$
\left|\Psi_{v}(r, t)\right\rangle=\mathcal{G}(t) \exp \left(-i \widetilde{\mathcal{E}}_{v} t\left|\Phi_{v}(r)\right\rangle\right.
$$

the cyclic solutions can be written in the form

$$
\left|\widetilde{\Psi}_{\nu}(r, t)\right\rangle=\left(\begin{array}{cc}
\exp \left(-i\left(\widetilde{\mathcal{E}}_{1}^{v}+\frac{\omega}{2}\right) t\right) & 0 \\
0 & \exp \left(-i\left(\widetilde{\mathcal{E}}_{2}^{v}-\frac{\omega}{2}\right) t\right)
\end{array}\right)\left|\Phi_{v}(r)\right\rangle,
$$

note that the superscript and subscript $v$ here and below indicates the dependence of $\mathcal{E}$ on $v$.

Let us present the time-independent Hamiltonian (7) and the time-dependent one (14) in forms (7) and (6); suitable for describing the motion of a spin 1/2-particle in the inhomogeneous magnetic field [14]:

$$
\widetilde{H}(r)=\left(p_{r}^{2}+q(r)\right) \hat{I}+\widetilde{\Omega}(r)\left(\begin{array}{cc}
\cos \widetilde{\theta}(r) & \sin \widetilde{\theta}(r) \\
\sin \widetilde{\theta}(r) & -\cos \widetilde{\theta}(r)
\end{array}\right),
$$

where $\widetilde{\mathbf{B}}(r)$ is expressed as

$$
\widetilde{\mathbf{B}}(r)=\widetilde{\Omega}(r)(\sin \widetilde{\theta}(r), 0, \cos \widetilde{\theta}(r)), \quad \widetilde{\Omega}(r)=\sqrt{B_{3}^{2}(r)+B_{1}^{2}(r)},
$$

The time-dependent Hamiltonian (14) can be rewritten in the form (6)

$$
H(t, r)=\left(p_{r}^{2}+q(r)\right) \hat{I}+\Omega(r)\left(\begin{array}{cc}
\cos \theta(r) & \sin \theta(r) \exp (-i \omega t) \\
\sin \theta(r) \exp (i \omega t) & -\cos \theta(r)
\end{array}\right)
$$

for a spin 1/2-particle in nonhomogeneous magnetic field $\mathbf{B}(t, r)$ precessing around $0 z$ axis

$$
\mathbf{B}(t, r)=\Omega(r)(\sin \theta(r) \cos (\omega t), \sin \theta(r) \sin (\omega t), \cos \theta(r)),
$$

where

$$
\begin{gathered}
\Omega(r)=\sqrt{\left(B_{3}(r)+\omega / 2\right)^{2}+B_{1}(r)^{2}}, \\
\sin \theta(r)=\frac{B_{1}(r)}{\Omega(r)}, \cos \theta(r)=\frac{B_{3}(r)+\omega / 2}{\Omega(r)} .
\end{gathered}
$$

By means of $\mathcal{G}(t)$ transformation (13) the Hamiltonian (17) turns to the time-dependent Hamiltonian written in terms of the components of the time-independent field

$$
H(t, r)=\left(p_{r}^{2}+q(r)\right) \hat{I}+\widetilde{\Omega}(r)\left(\begin{array}{cc}
\cos \widetilde{\theta}(r)+\omega / 2 \widetilde{\Omega}(r) & \sin \widetilde{\theta}(r) \exp (-i \omega t) \\
\sin \widetilde{\theta}(r) \exp (+i \omega t) & -\cos \widetilde{\theta}(r)-\omega / 2 \widetilde{\Omega}(r)
\end{array}\right)
$$


The comparison of (19) and (21) gives us

$$
\begin{gathered}
\Omega(r)=\widetilde{\Omega}(r)\left(1+\frac{\omega^{2}}{4 \widetilde{\Omega}^{2}(r)}+\frac{\omega}{\widetilde{\Omega}(r)} \cos \widetilde{\theta}(r)\right)^{1 / 2}, \\
\sin \theta(r)=\frac{\widetilde{\Omega}(r) \sin \widetilde{\theta}(r)}{\Omega(r)}, \cos \theta(r)=\frac{\widetilde{\Omega}(r) \cos \widetilde{\theta}(r)}{\Omega(r)}+\frac{\omega}{\Omega(r)} .
\end{gathered}
$$

As a result of the unitary transformation specified by $\mathcal{G}(t)$, the $B_{2}(t, r)$ - component of the vector $\mathbf{B}(t, r)$ arises.

If $\mathcal{G}(t)$ is chosen as an operator of rotation around $y$-axis

$$
\mathcal{G}_{2}(t)=\exp \left(-i \hat{\sigma}_{2} \frac{\omega_{2} t}{2}\right)=\left(\begin{array}{cc}
\cos \left(\frac{\omega_{2} t}{2}\right) & -\sin \left(\frac{\omega_{2} t}{2}\right) \\
\sin \left(\frac{\omega_{2} t}{2}\right) & \cos \left(\frac{\omega_{2} t}{2}\right)
\end{array}\right),
$$

then in accordance with (9) the time-independent Hamiltonian (7) turns to the time-dependent Hamiltonian $H(t)$ in the form

$$
H(r, t)=\left(p_{r}^{2}+q(r)\right) \hat{I}+\widetilde{\Omega}(r)\left(\begin{array}{cc}
C(r) c-S(r) s & S(r) c+C(r) s-\frac{i \omega_{2}}{2 \widetilde{\Omega}(r)} \\
S(r) c+C(r) s+\frac{i \omega_{2}}{2 \widetilde{\Omega}(r)} & -(C(r) c-S(r) s)
\end{array}\right)
$$

where $c=\cos \omega_{2} t, s=\sin \omega_{2} t$ and $C=\cos \widetilde{\theta}(r), S=\sin \widetilde{\theta}(r)$ and can be used in the description of the spin- $1 / 2$ particle in a time-dependent space-inhomogeneous magnetic field precessing around $0 y$ axis. In accordance with identity (15) the recurrent solutions of equation (1) are immediately written as

$$
\left|\Psi_{v}(r, t)\right\rangle=\left(\begin{array}{cc}
\cos \left(\omega_{2} t / 2\right) \exp \left(-i \widetilde{\mathcal{E}}_{1}^{v} t\right) & -\sin \left(\omega_{2} t / 2\right) \exp \left(-i \widetilde{\mathcal{E}}_{2}^{v} t\right) \\
\sin \left(\omega_{2} t / 2\right) \exp \left(-i \widetilde{\mathcal{E}}_{1}^{v} t\right) & \cos \left(\omega_{2} t / 2\right) \exp \left(-i \widetilde{\mathcal{E}}_{2}^{v} t\right)
\end{array}\right)\left|\Phi_{v}(r)\right\rangle
$$

and in one period

$$
\left|\Psi_{v}(r,(t+T))\right\rangle=-\left(\begin{array}{cc}
\exp \left(-i \widetilde{\mathcal{E}}_{1}^{v} T\right) & 0 \\
0 & \exp \left(-i \widetilde{\mathcal{E}}_{2}^{v} T\right)
\end{array}\right)\left|\Psi_{\nu}(r, t)\right\rangle .
$$

When there is no dependence on the space variable, the time-dependent Hamiltonian (24) and the corresponding cyclic solutions (25) become

$$
\begin{gathered}
H(t)=\widetilde{\Omega}\left(\begin{array}{cc}
\cos \widetilde{\theta} \cos \omega_{2} t-\sin \widetilde{\theta} \sin \omega_{2} t & \sin \widetilde{\theta} \cos \omega_{2} t+\cos \widetilde{\theta} \sin \omega_{2} t-\frac{i \omega_{2}}{2 \widetilde{\Omega}} \\
\sin \widetilde{\theta} \cos \omega_{2} t+\cos \widetilde{\theta} \sin \omega_{2} t+\frac{i \omega_{2}}{2 \widetilde{\Omega}} & -\left(\cos \widetilde{\theta} \cos \omega_{2} t-\sin \widetilde{\theta} \sin \omega_{2} t\right)
\end{array}\right) \\
\left|\Psi_{1}(t)\right\rangle=\cos \left(\omega_{2} t / 2\right) \exp \left(-i \widetilde{\mathcal{E}}_{1} t\right) \cos \widetilde{\theta} / 2- \\
\sin \left(\omega_{2} t / 2\right) \exp \left(-i \widetilde{\mathcal{E}}_{2} t\right) \sin \widetilde{\theta} / 2 \sin \left(\omega_{2} t / 2\right) \exp \left(-i \widetilde{\mathcal{E}}_{1} t\right) \cos \widetilde{\theta} / 2 \\
\left.+\cos \left(\omega_{2} t / 2\right) \exp \left(-i \widetilde{\mathcal{E}}_{2} t\right) \sin \widetilde{\theta} / 2\right) \\
\mid \Psi_{2}(t)>=-\cos \left(\omega_{2} t / 2\right) \exp \left(-i \widetilde{\mathcal{E}}_{1} t\right) \sin \widetilde{\theta} / 2-
\end{gathered}
$$




$$
\begin{gathered}
\sin \left(\omega_{2} t / 2\right) \exp \left(-i \widetilde{\mathcal{E}}_{2} t\right) \cos \widetilde{\theta} / 2-\sin \left(\omega_{2} t / 2\right) \exp \left(-i \widetilde{\mathcal{E}}_{1} t\right) \sin \tilde{\theta} / 2+ \\
\cos \left(\omega_{2} t / 2\right) \exp \left(-i \widetilde{\mathcal{E}}_{2} t\right) \cos \widetilde{\theta} / 2 .
\end{gathered}
$$

This problem is identical to the motion of a spin- $1 / 2$ in the uniformly rotating magnetic field in the $(x, z)$-plane.

Let $\mathcal{G}(t)$ be chosen as an operator of rotation around $x$-axis

$$
\mathcal{G}(t)=\left(\begin{array}{cc}
\cos \left(\omega_{1} t / 2\right) & -i \sin \left(\omega_{1} t / 2\right) \\
-i \sin \left(\omega_{1} t / 2\right) & \cos \left(\omega_{1} t / 2\right)
\end{array}\right)
$$

By an analogy with the previous cases, the time-independent Hamiltonian (7) is transformed into the time-dependent one and can be represented in the form for a spin- $1 / 2$ particle in the space-inhomogeneous magnetic field precessing around $0 x$ axis:

$$
\begin{gathered}
H(r, t)=\left(p_{r}^{2}+q(r)\right) \hat{I}+ \\
\widetilde{\Omega}(r)\left(\begin{array}{cc}
\cos \widetilde{\theta}(r) \cos \left(\omega_{1} t\right) & \sin \widetilde{\theta}(r)-\frac{\omega_{1}}{2 \widetilde{\Omega}(r)}+i \cos \widetilde{\theta}(r) \sin \left(\omega_{1} t\right) \\
\sin \widetilde{\theta}(r)-\frac{\omega_{1}}{2 \widetilde{\Omega}(r)}-i \cos \widetilde{\theta}(r) \sin \left(\omega_{1} t\right) & -\cos \widetilde{\theta}(r) \cos \left(\omega_{1} t\right)
\end{array}\right) .
\end{gathered}
$$

The cyclic solutions of (1) with account of (15) are written as

$$
\left|\Psi_{v}(r, t)\right\rangle=\left(\begin{array}{cc}
\cos \left(\omega_{1} t / 2\right) \exp \left(-i \widetilde{\mathcal{E}}_{1}^{v} t\right) & -i \sin \left(\omega_{1} t / 2\right) \exp \left(-i \widetilde{\mathcal{E}}_{2}^{v} t\right) \\
-i \sin \left(\omega_{1} t / 2\right) \exp \left(-i \widetilde{\mathcal{E}}_{1}^{v} t\right) & \cos \left(\omega_{1} t / 2\right) \exp \left(-i \widetilde{\mathcal{E}}_{2}^{v} t\right)
\end{array}\right)\left|\Phi_{\nu}(r)\right\rangle .
$$

Thus, by a different choice of transformation operators $\mathcal{G}_{i}(t), i=1,2,3$, three families of timedependent potential matrices with the corresponding cyclic solutions are generated in a closed form from one family of time-independent potential matrices. The obtained expressions turn into ones for a spin-1/2 particle evolving in a periodic time-dependent space-uniform magnetic field. It is evident that, in all the cases considered, the Hamiltonians (14), (24), (27) are $T$-periodic, $T=2 \pi / \omega$, and the solutions (16), (25) and (28) are $2 T=4 \pi / \omega$-periodic and change their sign after one period. As one can easily see, if $\mathcal{G}(t)$ is chosen to be $T$-periodic, e.g., $\mathcal{G}_{i}(t)=\exp \left(-i \hat{\sigma}_{i} \omega_{i} t\right)$, then the Hamiltonian becomes $T / 2=\pi / \omega$-periodic, and the solutions are $T$-periodic. In this case, the total phase is a multiple of $2 \pi, \beta_{v}=\widetilde{\mathcal{E}}_{v} T+2 \pi$. Clearly, a more general transformation can be taken: i) as a direct product of the $\mathcal{G}_{i}(t), \mathcal{G}(t)=\prod_{i=1}^{3} \mathcal{G}_{i}(t)$, ii) with a more complicated dependence of $\mathcal{G}$ on time [14].

\section{Construction of an Universal Set of Gates}

A method for obtaining a universal set of quantum gates with the use of the explicit form of evolution matrices for time-dependent Hamiltonians is presented in this section. Logical operators in a quantum computer are separated into the discrete set of time sequential basis quantum operations, quantum gates. Each gate performs a unitary transformation of a certain number of qubits. A qubit is an information carrier in the quantum computer and is an analog of the classical bit. One of the main conditions necessary for constructing the quantum computer is the existence of a universal set of one- and two-qubit quantum gates that can provide an arbitrary unitary transformation $\mathcal{U}(t)$ of an 
$n$-qubit quantum system (register) in the $2^{n}$-dimensional Hilbert space. We present a way to obtain a universal set of gates by using the time-dependent Hamiltonians considered above. Two-qubit gates are described by special $2 \times 2$ matrices, application of which leads to the interchange of the states, and form an universal set of quantum gates. All of these matrices can be obtained from the dynamic evolution of quantum system, which will provide quantum program with needed unitary matrices at each fixed moment.

Using the evolution operator (12) and cyclic solutions $\Psi_{1}(t), \Psi_{2}(t)$ we obtain an evolution matrix

$$
U(t)=\left(\begin{array}{ll}
u_{11}(t) & u_{12}(t) \\
u_{21}(t) & u_{22}(t)
\end{array}\right)
$$

where

$$
\begin{aligned}
& u_{11}\left(\omega_{2}, \widetilde{\mathcal{E}}_{1}^{v}, \widetilde{\mathcal{E}}_{2}^{v}, \widetilde{\theta}, t\right)=\cos \left(\omega_{2} t / 2\right) \exp \left(-i \widetilde{\mathcal{E}}_{1} t\right) \cos \widetilde{\theta} / 2-\sin \left(\omega_{2} t / 2\right) \exp \left(-i \widetilde{\mathcal{E}}_{2} t\right) \sin \tilde{\theta} / 2, \\
& u_{12}\left(\omega_{2}, \widetilde{\mathcal{E}}_{1}^{v}, \widetilde{\mathcal{E}}_{2}^{v}, \widetilde{\theta}, t\right)=-\cos \left(\omega_{2} t / 2\right) \exp \left(-i \widetilde{\mathcal{E}}_{1} t\right) \sin \widetilde{\theta} / 2-\sin \left(\omega_{2} t / 2\right) \exp \left(-i \widetilde{\mathcal{E}}_{2} t\right) \cos \widetilde{\theta} / 2, \\
& u_{21}\left(\omega_{2}, \widetilde{\mathcal{E}}_{1}^{v}, \widetilde{\mathcal{E}}_{2}^{v}, \widetilde{\theta}, t\right)=\sin \left(\omega_{2} t / 2\right) \exp \left(-i \widetilde{\mathcal{E}}_{1} t\right) \cos \widetilde{\theta} / 2+\cos \left(\omega_{2} t / 2\right) \exp \left(-i \widetilde{\mathcal{E}}_{2} t\right) \sin \tilde{\theta} / 2, \\
& u_{22}\left(\omega_{2}, \widetilde{\mathcal{E}}_{1}^{v}, \widetilde{\mathcal{E}}_{2}^{v}, \widetilde{\theta}, t\right)=-\sin \left(\omega_{2} t / 2\right) \exp \left(-i \widetilde{\mathcal{E}}_{1} t\right) \sin \widetilde{\theta} / 2+\cos \left(\omega_{2} t / 2\right) \exp \left(-i \widetilde{\mathcal{E}}_{2} t\right) \cos \tilde{\theta} / 2 .
\end{aligned}
$$

Let us introduce the following notations $\frac{\omega_{2}}{2}=\alpha, \widetilde{\mathcal{E}}_{1}^{v}=-\widetilde{\mathcal{E}}_{2}^{v}=\lambda, \widetilde{\theta} / 2=\beta$

$$
U(\alpha, \beta, \lambda ; t)=\left(\begin{array}{ll}
u_{11}(\alpha, \beta, \lambda ; t) & u_{12}(\alpha, \beta, \lambda ; t) \\
u_{21}(\alpha, \beta, \lambda ; t) & u_{22}(\alpha, \beta, \lambda ; t)
\end{array}\right) \text {, }
$$

where

$$
\begin{aligned}
& u_{11}(\alpha, \beta, \lambda ; t)=\cos (\alpha t) \exp (-i \lambda t) \cos (\beta)-\sin (\alpha t) \exp (i \lambda t) \sin (\beta), \\
& u_{12}(\alpha, \beta, \lambda ; t)=-\cos (\alpha t) \exp (-i \lambda t) \sin (\beta)-\sin (\alpha t) \exp (i \lambda t) \cos (\beta), \\
& u_{21}(\alpha, \beta, \lambda ; t)=\sin (\alpha t) \exp (-i \lambda t) \cos (\beta)+\cos (\alpha t) \exp (i \lambda t) \sin (\beta), \\
& u_{22}(\alpha, \beta, \lambda ; t)=-\sin (\alpha t) \exp (-i \lambda t) \sin (\beta)+\cos (\alpha t) \exp (i \lambda t) \sin (\beta)
\end{aligned}
$$

and rewrite the given evolution operator as a product of three unitary matrices

$$
U(t)=\left(\begin{array}{cc}
\cos (\alpha t) & \sin (\alpha t) \\
\sin (\alpha t) & \cos (\alpha t)
\end{array}\right)\left(\begin{array}{cc}
\exp (-i \lambda t) & 0 \\
0 & \exp (i \lambda t)
\end{array}\right)\left(\begin{array}{cc}
\cos (\beta) & -\sin (\beta) \\
\sin (\beta) & \cos (\beta)
\end{array}\right) .
$$

Evidently, $U(\alpha, \beta, \lambda, t)$ generates all matrices from $S U(2)$. The action of the right matrix on the basis state $|0\rangle$ or $|1\rangle$ yields all coherent states, which by using a diagonal matrix of the given form of $U(t)$ can be rotated around $y$ axis in a controlled way.

For any $\alpha, \beta, \gamma$, at $t=0$ from (30) we obtain the gate

$$
U(\alpha, \beta, \gamma ; t)=\left(\begin{array}{cc}
1 & -1 \\
1 & 1
\end{array}\right)
$$


To obtain the Hadamard gate

$$
H=\frac{1}{\sqrt{2}}\left(\begin{array}{cc}
1 & 1 \\
1 & -1
\end{array}\right)
$$

we multiply $U(\alpha, \beta, \gamma ; t)$ from the left on the gate

$$
N O T=\left(\begin{array}{ll}
0 & 1 \\
1 & 0
\end{array}\right) .
$$

At $t=\frac{\pi}{2 \alpha}$ and $\lambda=0$, we have $U\left(\alpha, \beta, 0 ; \frac{\pi}{2 \alpha}\right)=N O T$ and therefore the Hadamard gate $H$ is a result of sequence of gates

$$
H=U\left(\alpha, \beta, 0 ; \frac{\pi}{2 \alpha}\right) U(\alpha, \beta, \gamma ; 0) .
$$

The single qubit gate $U$ can be obtained as a product of unitary matrices

$$
U=U\left(\alpha_{k}, \beta_{k}, \gamma_{k} ; t_{k}\right) U\left(\alpha_{k-1}, \beta_{k-1}, \gamma_{k-1} ; t_{k-1}\right) \ldots U\left(\alpha_{0}, \beta_{0}, \gamma_{0} ; t_{0}=0\right) .
$$

Here $\alpha_{j}, \beta_{j}, \gamma_{j}, j=0, \ldots, k$ are any real parameters and the parameters of discrete time $t_{j}, j=$ $0, \ldots, k$ satisfy the conditions $0=t_{0}<t_{1}<\ldots<t_{k}$.. Note that in our approach, the effect of geometric phase is naturally taken into account in the construction of single qubit gates.

Indeed, for the periodic evolution matrix with the period $T=2 \pi m$, the state changes sign when $\alpha_{j} t_{j}=2 \pi m$ or $\gamma_{j} t_{j}=2 \pi m$. In this way, we arrive at the binary representation of the numbers from 0 to $2^{n}-1$ :

$$
\begin{gathered}
(H \otimes H \otimes \cdots \otimes H)|00 \ldots 0\rangle= \\
=U\left(\alpha, \beta, 0 ; \frac{\pi}{2 \alpha}+2 n \pi\right) U(\alpha, \beta, \gamma ; 2 n \pi) \otimes \cdots \otimes U\left(\alpha, \beta, 0 ; \frac{\pi}{2 \alpha}\right) U(\alpha, \beta, \gamma ; 0)|00 \ldots 0\rangle= \\
=\frac{1}{\sqrt{2^{n}}}((|0\rangle+|1\rangle) \otimes(|0\rangle+|1\rangle) \otimes \cdots \otimes(|0\rangle+|1\rangle))=\frac{1}{\sqrt{2^{n}}} \sum_{x=0}^{2^{n}-1}|x\rangle .
\end{gathered}
$$

It should be noted that, for the presented sequence of gates, the obtained states $\frac{1}{\sqrt{2^{n}}} \sum_{x=0}^{2^{n}-1}|x\rangle$ are not unique [4].

Let us now turn to a construction of two-qubit gates. Note that the operator $S W A P: C^{2} \otimes C^{2} \rightarrow$ $C^{2} \otimes C^{2}$, acting on tensor product of vectors $S W A P(u \otimes v)=v \otimes u$ and taking in the standard basis the form

$$
S W A P=\left(\begin{array}{llll}
1 & 0 & 0 & 0 \\
0 & 0 & 1 & 0 \\
0 & 1 & 0 & 0 \\
0 & 0 & 0 & 1
\end{array}\right)
$$

is not an entangled operator, and the operator, $b u r=\left(\begin{array}{llll}0 & 1 & 0 & 0 \\ 1 & 0 & 0 & 0 \\ 0 & 0 & 1 & 0 \\ 0 & 0 & 0 & 1\end{array}\right)$ (obtained from the Burau representation [4]) as well as the operator CNOT is the entangled operator but despite this fact all these operators have the same normal Jordan's form. 
The Hermitian operator of the form $A=\left(\begin{array}{cccc}0 & 0 & 0 & 0 \\ 0 & 0 & 1 & 0 \\ 0 & 1 & 0 & 0 \\ 0 & 0 & 0 & 0\end{array}\right)$ has the rank 2 and commutes with the operators of the form

$$
\text { bur }=\left(\begin{array}{cccc}
x_{11} & 0 & 0 & x_{14} \\
0 & a & b & 0 \\
0 & b & a & 0 \\
x_{41} & 0 & 0 & x_{44}
\end{array}\right)
$$

and the operator $\exp (-i A t)$ is an entangled one. Indeed,

$$
\exp (-i A t)=\left(\begin{array}{cccc}
1 & 0 & 0 & 0 \\
0 & \cos (t) & -i \sin (t) & 0 \\
0 & -i \sin (t) & \cos (t) & 0 \\
0 & 0 & 0 & 1
\end{array}\right)
$$

at $t=\pi / 4$ takes the form

$$
\exp (-i A \pi / 4)=\left(\begin{array}{cccc}
1 & 0 & 0 & 0 \\
0 & \sqrt{2} / 2 & -i \sqrt{2} / 2 & 0 \\
0 & -i \sqrt{2} / 2 & \sqrt{2} / 2 & 0 \\
0 & 0 & 0 & 1
\end{array}\right)
$$

The universal CNOT consisting from $\exp (-i A \pi / 4)$ and one-qubit gates looks as follows

$$
\begin{gathered}
C N O T=\left(X_{1} \otimes 1\right)(1 \otimes 1)\left(X_{2} \otimes 1\right)\left(1 \otimes Y_{2}\right) \times \\
\times \exp (-i A \pi / 4)\left(X_{3} \otimes 1+1 \otimes Y_{3}\right) \exp (-i A \pi / 4)\left(X_{4} \otimes 1+1 \otimes Y_{4}\right)\left(1 \otimes Y_{5}\right)\left(X_{5} \otimes 1\right),
\end{gathered}
$$

where

$$
\begin{gathered}
X_{1}=\sqrt{2} / 2\left(\begin{array}{cc}
1 & -1 \\
1 & 1
\end{array}\right), X_{2}=\sqrt{2} / 2\left(\begin{array}{cc}
-i & 1 \\
i & 1
\end{array}\right), Y_{2}=\sqrt{2} / 2\left(\begin{array}{cc}
1 & i \\
i & -i
\end{array}\right), \\
X_{3}=\left(\begin{array}{cc}
0 & -i \\
i & 0
\end{array}\right), Y_{3}=1=\left(\begin{array}{cc}
1 & 0 \\
0 & 1
\end{array}\right), X_{4}=1 / 2\left(\begin{array}{cc}
1-i & -1-i \\
-1-i & 1-i
\end{array}\right), \\
Y_{4}=\sqrt{2} / 2\left(\begin{array}{cc}
1 & -1 \\
-i & 1
\end{array}\right), X_{5}=\sqrt{2} / 2\left(\begin{array}{cc}
1 & 1 \\
-1 & 1
\end{array}\right), Y_{5}=\sqrt{2} / 2\left(\begin{array}{cc}
1 & 0 \\
0 & -i
\end{array}\right) .
\end{gathered}
$$

It is easy to show that if $H$ is a two-dimension diagonal time-independent Hamiltonian of the form

$$
h=\left(\begin{array}{ll}
a & 0 \\
0 & b
\end{array}\right),
$$

then the evolution operator of the matrix Schrödinger equation (1) with the Hamiltonian $H=$ $h \otimes 1+1 \otimes h+\epsilon A$ is expressed as follows

$$
U(t)=\left(e^{-i h t} \otimes e^{-i h t}\right) e^{-i A t},
$$


where $\epsilon \in\{0,1\}$.

Indeed, from this given property of operator $A$ follows that the matrix

$$
h \otimes 1+1 \otimes h=\left(\begin{array}{cccc}
2 a & 0 & 0 & 0 \\
0 & a+b & 0 & 0 \\
0 & 0 & a+b & 0 \\
0 & 0 & 0 & 2 b
\end{array}\right)
$$

commutes with $A$ and, as a consequence, $U(t)=e^{-i(h \otimes 1+1 \otimes h+A) t}=\left(e^{-i h t} \otimes e^{-i h t}\right) e^{-i A t}$. The Pauli matrix $\sigma_{3}=\left(\begin{array}{cc}1 & 0 \\ 0 & -1\end{array}\right)$ satisfies the above condition. Substituting $h=\sigma_{3}$ we get an entangled operator

$$
U(t)=\left(\begin{array}{cccc}
e^{i t} & 0 & 0 & 0 \\
0 & \cos (t) & -i \sin (t) & 0 \\
0 & -i \sin (t) & \cos (t) & 0 \\
0 & 0 & 0 & e^{-i t}
\end{array}\right)
$$

It is interesting to note that even in the case when the initial Hamiltonian does not give an entangled operator, after a suitable choice of the gauge transformation one can get Hamiltonian giving entanglement. In addition, the geometric phase is obtained from the cyclic evolution of states (see [14]).

\section{Conclusion}

Having an explicit form for the evolution matrix of a 2-level system it is not difficult to construct a register with $N$ qubits. An universal 1-qubit logic gate can be constructed from the time evolution matrices which we obtain in a closed analytic form.

If a quantum system undergoes a cyclic evolution, the initial- and final-state vectors are related by a geometric phase factor.

We give an example of the quantum register and discuss a way of obtaining entanglement operator from mathematical point of view. It is known (see [4]) that the universal set of gates are given by $2 \times 2$ unitary operators and one unitary entangled operator $R$ : which acts on $C^{2} \otimes C^{2}$ and transforms decomposed tensor into not decomposed one. It means that there is a vector $|u v\rangle=|u\rangle|v\rangle \in C^{2} \otimes C^{2}$ such that $R|u v\rangle$ is not decomposed as a tensor product of two-qubits. In our case the entanglement operator will be obtained from two independent systems with the use of unitary gauge time-dependent transformations, which leads to time-dependent periodic operator and entanglement of states.

The method of construction of the universal set of quantum quantum gates, given here, is applicable for three level quantum systems and in monodromy approach to quantum computation [5], [7], where monodromy matrices play the role of geometric phase and it is possible to construct Hamiltonian of quantum system with prescribed properties [8]. In case, where the universal set of 
quantum gates cannot be constructed in explicit form there arises the problem of controllability of quantum system [6].

Acknowledgement. This work was supported by the joint grant of Volkswagen Foundation and Shota Rustaveli National Science Foundation of Georgia (Ref. 93562 \& \# 04/48).

\section{References}

[1] Aharonov, Y., Anandan, J. Phase change during a cyclic quantum evolution. Phys. Rev. Lett. 58, p. 1593, 1987.

[2] Berry, M. Quantal phase factors accompanying adiabatic changes. Proc. R. Soc. London, Ser. A 392, 45, 1984.

[3] Bohm, A., Mostafazadeh, A., Koizumi, H., Niu, Q., Zwanziger J. The Geometric Phase in Quantum Systems. Springer, 2003.

[4] Giorgadze, G. Geometry of Quantum Computations. Nova Publishers, 2013.

[5] Giorgadze, G. Monodromy matrices as universal set of quantum gates and dynamics of cold trapped ions. Tbilisi Mathematical Journal, 13, 2, 187-206, 2020.

[6] Giorgadze, G. Control of quantum processing based on the three-level quantum system. In "Optimal control and differential games", Materials of the international conference dedicated to L.Pontryagin, Moscow, 103-105, 2018.

[7] Giorgadze, G. Monodromic approach to quantum computing. Int. J. Modern Physics B 16, 30, 4593-4605, 2002.

[8] Giorgadze, G., Khimshiashvili, G. On Shrödinger equations of Okubo type. J. Dyn. Cont. Syst. 10, 2, 171-186, 2004.

[9] Kuznezov, D., Bulgac, A. Canonical ensembles from chaos II: Constrained dynamical systems. Ann. Phys. 214, 180-218, 1992.

[10] Landau, L. D. A Theory of Energy Transfer on Collisions. Phys. Z. Sov. 1, p. 88, 1932.

[11] Mead, C. A., Trular, D.G. On the determination of Born-Oppenheimer nuclear motion wave functions including complications due to conical intersections and identical nuclei. J. Chem. Phys. 70, p. 2284, 1979 .

[12] Nielsen M., Chuang, I. Quantum Computation and Quantum Information. Cambridge Univ. Press, 2000.

[13] Paul, W., Raether, M. Das elektrische Massenfilter. Z. Phys. 140, 262-273, 1955.

[14] Suzko A.A.; Giorgadze, G. Exactly solvable time-dependent models in quantum mechanics and their applications. Physics of Particles and Nuclei. 39, 4, 578-596, 2008. 
[15] Zanardi P., Rasetti M. Holonomic quantum computation. Phys. Lett. A, 264, 94-99, 1999.

[16] Zener, C. Non-adiabatic crossing of energy levels. Proc. R. Soc. London, Ser. A, 137, p. 696, 1932. 